\title{
Elevated Neutrophil Gelatinase Lipocalin Levels Are Associated With Increased Oxidative Stress in Hemodialysis Patients
}

\author{
Adam Nevo a, f, g, Zaher Armalyb, f, Amir Abd El Kadirc , Amos Douvdevanid, \\ David Tovbin ${ }^{\mathrm{e}}$
}

\begin{abstract}
Background: Administration of intravenous iron is an essential treatment of anemia in hemodialysis patients, but it may lead to oxidative stress and increased morbidity and mortality. There is evidence that neutrophil gelatinase-associated lipocalin (NGAL) is protective against oxidative stress and thus the aim of the present study was to investigate the relationship between plasma NGAL and advanced oxidative protein products (AOPP) in hemodialysis patients treated with intravenous iron.
\end{abstract}

Methods: In a prospective study, 47 hemodialysis patients (mean age 63 years, $\mathrm{SD}=13.6$; $40 \%$ women) were enrolled from two separate hospitals. Oxidative stress was induced by an intravenous administration of $100 \mathrm{mg}$ iron saccharate $0.5 \mathrm{~h}$ after the start of dialysis. Blood samples were drawn at the beginning of the dialysis, $0.5 \mathrm{~h}$ after iron administration and at the end of dialysis. NGAL levels were measured from the first blood sample, AOPP levels were measured from all blood samples.

Results: Our results showed that higher NGAL and AOPP levels at the beginning of the dialysis, prior to iron administration, significantly predicted higher levels of AOPP toward the end of dialysis, $(\beta=0.355$, $\mathrm{SE}=0.054, \mathrm{P}=0.035 ; \beta=0.297, \mathrm{SE}=0.159, \mathrm{P}=0.043$, respectively)

Conclusions: Our results suggest that higher level of NGAL is a risk factor for oxidative stress, as measured by AOPP levels, in dialysis patients receiving intravenous iron. Our findings could identify dialysis patients who are at higher risk from iron supplementation via

Manuscript submitted January 13, 2018, accepted January 29, 2018

${ }^{a}$ Cardiothoracic Division, Department of Surgery, Duke University Medical Center, Durham, NC, USA

${ }^{b}$ Department of Nephrology, EMMS Nazareth -The Nazareth Hospital, Nazareth, Galilee Medical School-Bar Ilan University, Safed, Israel

'Soroka Medical Center, Ben-Gurion University of the Negev, Beer-Sheva, Israel

${ }^{\mathrm{d}}$ Department of Nephrology, Soroka Medical Center, Ben-Gurion University of the Negev, Beer-Sheva, Israel

'Department of Nephrology, Haemek Medical Center, Afula, Israel

fThese authors contributed equally to this manuscript.

'Corresponding Author: Adam Nevo, Cardiothoracic Division, Department of Surgery, Duke University Medical Center, MSRB 1, Durham, NC 27710 , USA.Email: adn19@duke.edu

doi: https://doi.org/10.14740/jocmr3360w measurement of NGAL levels.

Keywords: NGAL; Hemodialysis; Oxidative stress; AOPP

\section{Introduction}

Oxidative stress contributes significantly to the morbidity and mortality of hemodialysis (HD) patients [1,2]. Oxidative stress is related to inflammation, as there is a positive correlation between markers of oxidative stress, such as advanced oxidation protein products (AOPP) and inflammatory markers such as C-reactive protein [3-5].

Anemia is a major cause of morbidity and mortality in HD patients [6]. Iron deficiency $[7,8]$ is a common cause of anemia in HD patients and has been linked to cardiovascular risk [9] even without a concomitant diagnosis of anemia. Consequently, one of the more prevalent treatments for anemia in HD patients is the administration of intravenous (IV) iron [10]. However, IV iron may be a double-edged sword as it has been associated with higher levels of AOPP levels [11] and increased morbidity and mortality [12].

AOPP also plays a role in acute kidney injury (AKI) [13], which is important to HD patients as episodes of AKI and decreased kidney perfusion during HD have been shown to reduce residual renal function, a factor in HD patients' clinical outcome $[14,15]$.

To better understand the relationship between oxidative stress, inflammation and HD, a great deal of research has been conducted to find endogenous mediators of these interactions.

One such potential target is neutrophil gelatinase associated lipocalin (NGAL). Produced and secreted by neutrophils, liver cells and kidney tubules, NGAL binds bacteria secreted iron-binding siderophores, particularly enterochelin, thus preventing bacteria from utilizing its iron for their growth, functioning as an endogenous antimicrobial [16].

The levels of NGAL are relatively low in healthy subjects [17], but rise exponentially in the tubules and serum in response to AKI [18, 19], ischemia [19] and after IV iron administration [20]. This is possibly due to the interaction between NGAL and the epidermal growth factor receptor [21].

This connection has led to the exploration of NGAL as a diagnostic biomarker and indeed NGAL has been shown to be 
superior to creatinine as a marker for acute renal damage and renal disease $[17,22]$. NGAL levels have also shown promise in predicting which patients will require long-term hospitalization and HD [23].

Beyond a potential role for NGAL as a predictive biomarker, there is also evidence of a protective role for NGAL against oxidative stress. Cell lines that were engineered to produce high levels of NGAL also produced powerful antioxidants [24]. In a mouse model, direct infusion of NGAL induced tubular regeneration after ischemia and reperfusion [25]; in another study, NGAL knockout mice showed far less tubular proliferation after nephron reduction [21].

This body of evidence has led us to hypothesize that NGAL might be protective against oxidative stress in HD patients. In this study, we aimed to elucidate the role NGAL plays in oxidative stress in HD patients receiving IV iron. To do this we examined the relationship between pre-dialysis NGAL levels and AOPP levels following IV iron administration.

\section{Methods}

\section{Patients}

The study included 47 HD patients: 35 patients from EMMS Nazareth Hospital and 12 patients from Soroka Medical Center. Exclusion criteria were: 1) receiving iron supplementation or blood transfusions in the 3 weeks prior to the study; 2) having acute infectious or systemic disease, active liver disease, acute myocardial infarction, cerebrovascular disease or a known diagnosis of hepatitis B, C or HIV. Mean age of patients was $63 \pm 14$ years, mean months on dialysis was $76 \pm 42 ; 60 \%$ $(\mathrm{n}=28)$ were male, $77 \%(\mathrm{n}=36)$ were married, $47 \%(\mathrm{n}=22)$ were Muslim, 28\% $(n=13)$ Christian and the rest Jewish. All demographic data were taken from patients' medical records. All procedures were approved by the Soroka Ethical Committee and followed the Helsinki guidelines. All patients signed a statement of informed consent in their native tongue, after it was fully explained to them in their native tongue.

\section{Study protocol}

HD patients routinely undergo hemodialysis three times a week, $4 \mathrm{~h}$ per session. Hemodialysis was carried out on Gambro dialyzer machines with FX80 dialysis coils. Heparin was given IV to all patients via the arterial line. Half an hour after the beginning of dialysis they were treated with $100 \mathrm{mg}$ of iron saccharate (Venofer, Luitpold Pharmaceuticals, Inc. Shirley, $\mathrm{NY}$ ) in $150 \mathrm{~mL} 0.9 \%$ saline. IV iron was administered in accordance with the recommendations of the American National Kidney Foundation. All patients were asked to avoid fat-containing foods the day before and during the study to prevent derangements of plasma AOPP levels.

Blood was drawn from the arterial line at three time points: the first, at the start of HD; the second, $1 \mathrm{~h}$ into the dialysis $(0.5 \mathrm{~h}$ after iron administration); and the third, after $4 \mathrm{~h}$ at the end of dialysis (3.5 $\mathrm{h}$ after iron administration). Notably, due to a late change

\section{AOPP Levels Change Through HD}

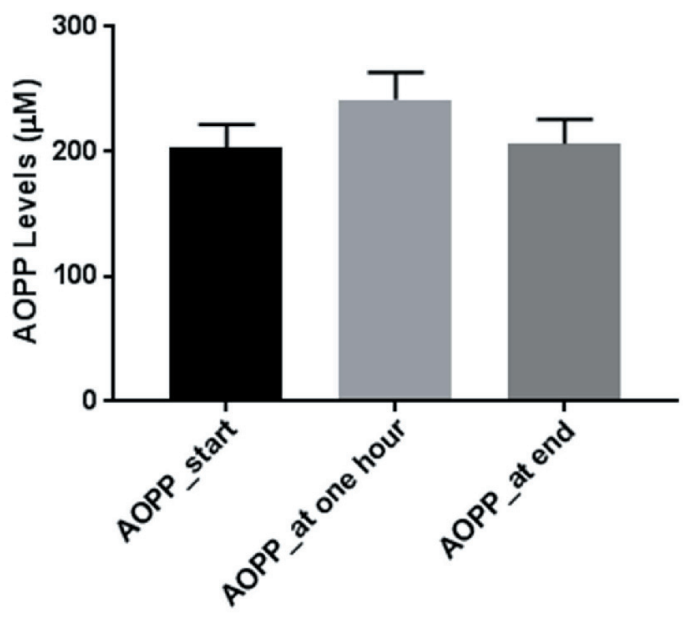

Figure 1. Mean levels of AOPP at the beginning of dialysis, before iron administration, $1 \mathrm{~h}$ into the dialysis and $0.5 \mathrm{~h}$ after iron administration, and at the end of dialysis, $3.5 \mathrm{~h}$ after iron administration.

in the study's protocol, only the patients from EMMS Nazareth Hospital had blood drawn at the second time point. All blood samples were analyzed for levels of hemoglobin $(\mathrm{Hb})$ and AOPP. NGAL levels were measured only from the first blood sample.

\section{Laboratory measurements}

$\mathrm{Hb}$ levels before and after dialysis, were measured by the Hematological Lab in the Soroka Medical Center. In order to measure AOPP levels, blood samples drawn into pre-heparinized blood tubes were centrifuged for $10 \mathrm{~min}$ to obtain plasma. AOPP levels were then measured via spectrophotometer, using the method described by Witko-Sarsat [26], at $340 \mathrm{~nm}$. The method includes turning samples into chloramine-T equivalents by fivefold dilution of $200 \mu \mathrm{L}$ of plasma with $20 \mathrm{mmol} / \mathrm{L}$ of phosphate-buffered saline (PBS), at $\mathrm{pH} 7.4$ followed by the addition of $80 \mu \mathrm{L}$ of acetic acid to $800 \mu \mathrm{L}$ of the dilution. The spectrophotometer was calibrated before reading the samples using a blank with PBS and acetic acid. NGAL levels were measured using an ELISA kit supplied by BioPorto Diagnostics (Hellerup, Denmark) and preformed according to the protocol provided by the company.

\section{Statistical analysis}

Statistical analysis was done using SPSS statistics version 19.0. Demographic (age, marital status, gender, months of dialysis, Hb levels and levels of NGAL) comparisons between Soroka Medical Center and EMMS Nazareth Hospital patient groups, were done using the Mann-Whitney rank test and Fisher's exact test due to the small sample size from Soroka Medical Center. Normality was determined with the Shapiro-Wilk test of normality. A linear regression was used to test for the effect of NGAL on AOPP levels at the end of dialysis, $3.5 \mathrm{~h}$ 
Table 1. Descriptive Statistics for the Main Variables and Covariates $(n=47)$

\begin{tabular}{lllll} 
& Min & Max & Mean & SD \\
\hline Age (y) & 29 & 90 & 69 & 10.9 \\
Months of dialysis (m) & 18 & 229 & 76 & 42 \\
Hb (g/dL) & 8.8 & 14.1 & 11.6 & 1.08 \\
NGAL levels (ng/mL) & 602 & 2,046 & $1,188.5$ & 373.2 \\
AOPP at start of dialysis & 16.31 & 625.38 & 202.9 & 125.7 \\
AOPP at the 1 h mark & 29.75 & 502.88 & 241 & 129 \\
AOPP at end of dialysis & 15.69 & 596 & 206 & 126.1 \\
\hline
\end{tabular}

after iron administration, while controlling for baseline levels of AOPP, age, gender ( 1 = male; 2 = female $),$ Hb levels, and months of dialysis.

The datasets generated and/or analyzed during the current study are available from the corresponding author on reasonable request.

\section{Results}

\section{Circulatory AOPP and NGAL levels}

Figure 1 depicts AOPP levels in HD patients at the beginning of dialysis, $1 \mathrm{~h}$ into the dialysis ( $0.5 \mathrm{~h}$ after iron administration) and at the end of dialysis (3.5 $\mathrm{h}$ after iron administration). As can be seen, administration of iron caused an increase in blood AOPP after short time, but these levels decline to basal concentrations toward the end of hemodialysis course.

\section{Descriptive statistics}

Descriptive statistics are provided in Table 1. As can be seen in Table 2, there was significant variation between the EMMS Nazareth Hospital patients and the Soroka Medical Center patients in age and familial status. The patients in the EMMS

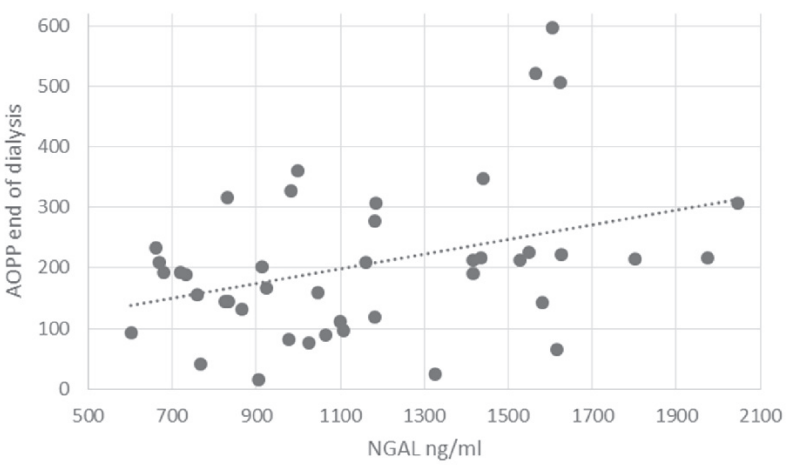

Figure 2. The relationship between NGAL levels and AOPP at end of dialysis.

Nazareth Hospital tended to be younger and more likely to be married than their counterparts in Soroka Medical Center.

\section{Regression analysis}

Shapiro-Wilk test for normality was run on the AOPP levels at the end of dialysis (statistic $=0.984, \mathrm{P}=0.807$ ). Linear regression results showed that higher NGAL and AOPP levels at the start of the dialysis, before iron administration, significantly predicted higher levels of AOPP at the end of dialysis, $3.5 \mathrm{~h}$ after iron administration, $(\beta=0.355, \mathrm{SE}=0.054, \mathrm{P}=0.035$; $\beta=0.297, \mathrm{SE}=0.159, \mathrm{P}=0.043$, respectively). The relationship between NGAL levels and AOPP at end of dialysis is shown in Figure 2. Gender was near significance in the model $(\beta=-0.248, \mathrm{SE}=35.451, \mathrm{P}=0.083)$. An additional regression analysis was done on part of the sample, where the dependent variable was AOPP levels $1 \mathrm{~h}$ into dialysis $(0.5 \mathrm{~h}$ after iron administration). NGAL levels did not significantly predict levels of AOPP $1 \mathrm{~h}$ into dialysis $(\beta=0.236, \mathrm{SE}=0.068, \mathrm{P}=0.222)$.

\section{Discussion}

Our results show that NGAL levels at the start of HD before IV

Table 2. Differences in Demographics and Laboratory Data Between the Patients From Soroka and the English Hospital

\begin{tabular}{llll} 
& Soroka & English Hospital & P (Fisher's exact test and Mann-Whitney) \\
\hline $\mathrm{n}$ & 12 & 35 & \\
Gender (men/women) & $6 / 6$ & $22 / 13$ & 0.5 \\
Married/not married & $7 / 5$ & $31 / 4$ & 0.003 \\
$\mathrm{Hb}(\mathrm{g} / \mathrm{dL})$ & 11.3 & 11.8 & 0.3 \\
Months dialysis $(\mathrm{m})$ & 74.4 & 76.9 & 0.5 \\
NGAL levels $(\mathrm{ng} / \mathrm{mL})$ & 1130.7 & 1208.3 & 0.43 \\
Age $(\mathrm{y})$ & 69 & 61 & 0.04 \\
AOPP levels at start & 209.1 & 200.8 & 0.354 \\
AOPP levels at end & 236.5 & 194.2 & 0.144 \\
\hline
\end{tabular}

English Hospital: EMMS Nazareth Hospital 
iron administration are positively correlated with AOPP levels at the end of HD and that the whole model significantly predicted $28 \%$ of the variance in AOPP levels at the end of the dialysis, while NGAL significantly predicted $8 \%$ of the variance. As higher levels of AOPP are indicative of oxidative stress, the current findings suggest that higher NGAL levels at the beginning of HD may be predictive of greater oxidative stress after IV iron administration. To our knowledge, the current study is the first to show an association between NGAL and oxidative stress in HD patients receiving IV iron.

Although our initial hypothesis was that NGAL would be protective against oxidative stress, as there is a large body of evidence that points to this direction. There are also studies that have suggested that in humans higher levels of NGAL are correlated with worse clinical outcomes. Indeed, NGAL has been linked to autosomal dominant polycystic kidney disease [27], progression of proteinuria [28], kidney graft rejection [29] and end stage renal disease [30].

Our results failed to show any predictive value of NGAL levels for the AOPP levels $0.5 \mathrm{~h}$ after iron administration. This may be explained by the smaller sample size $(n=35)$ for which AOPP levels at the $1 \mathrm{~h}$ mark were available. It is also possible that NGAL's effects take time to manifest and that $0.5 \mathrm{~h}$ is too small a period to measure the effects. The temporal relationship between NGAL and AOPP needs to be further explored in future studies.

Our study has a few limitations. First, the sample size is relatively small, mostly as a result of the small number of dialysis patients who fit the inclusion criteria in Israel. Even though we were able to show a significant effect of NGAL, it is important to confirm our findings with a larger sample. Second, as has been mentioned above, all patients were asked to adhere to a non-fat diet, it is possible that not all participants adhered to the required diet before the study. Future studies should try to either directly control the diet of the participants in the study or question the participants regarding their diet and control for this variable statistically.

In conclusion, while the role of NGAL in oxidative stress in HD patients has been controversial, our results support literature showing the potentially adverse effects of high levels of NGAL. We have shown that high NGAL level is a risk factor for oxidative stress in HD patients receiving IV iron. Since there is a large body of literature pointing towards the opposite, NGAL being protective from oxidative stress, there has been some interest in using NGAL as a treatment in HD patients. Our findings represent evidence that this strategy could prove to be harmful and should be avoided. Furthermore, since dialysis patients are often dependent on IV iron, our findings could help identify dialysis patients who are at higher risk from iron supplementation via measurement of their NGAL levels and help clinicians devise better treatment modalities for these patients, thus improving their outcomes during hemodialysis.

\section{Ethical Approval}

All procedures performed in studies involving human par- ticipants were in accordance with the ethical standards of the institutional and/or national research committee and with the 1964 Helsinki declaration and its later amendments or comparable ethical standards.

\section{Conflict of Interest}

On behalf of all authors, the corresponding author states that there is no conflict of interest.

\section{Grant Support}

This research received no specific grant from any funding agency in the public, commercial, or not-for-profit sectors.

\section{References}

1. Spittle M, Hoenich NA, Handelman G, Adhikarla R, Homel P, Levin NW. Oxidative stress and inflammation in hemodialysis patients; Improving Prognosis for Kidney Disorders. Springer. 2002. p. 45-52.

2. Locatelli F, Canaud B, Eckardt KU, Stenvinkel P, Wanner C, Zoccali C. Oxidative stress in end-stage renal disease: an emerging threat to patient outcome. Nephrol Dial Transplant. 2003;18(7):1272-1280.

3. Yang XB, Hou FF, Wu Q, Zhou H, Liu ZR, Yang Y, Zhang $\mathrm{X}$. [Increased levels of advanced oxidation protein products are associated with atherosclerosis in chronic kidney disease]. Zhonghua Nei Ke Za Zhi. 2005;44(5):342-346.

4. Handelman GJ, Walter MF, Adhikarla R, Gross J, Dallal GE, Levin NW, Blumberg JB. Elevated plasma F2-isoprostanes in patients on long-term hemodialysis. Kidney Int. 2001;59(5):1960-1966.

5. Nguyen-Khoa T, Massy ZA, De Bandt JP, Kebede M, Salama L, Lambrey G, Witko-Sarsat V, et al. Oxidative stress and haemodialysis: role of inflammation and duration of dialysis treatment. Nephrol Dial Transplant. 2001;16(2):335-340.

6. Madore F, Lowrie EG, Brugnara C, Lew NL, Lazarus JM, Bridges K, Owen WF. Anemia in hemodialysis patients: variables affecting this outcome predictor. J Am Soc Nephrol. 1997;8(12):1921-1929.

7. Horl WH. Clinical aspects of iron use in the anemia of kidney disease. J Am Soc Nephrol. 2007;18(2):382-393.

8. Van Wyck DB, Stivelman JC, Ruiz J, Kirlin LF, Katz MA, Ogden DA. Iron status in patients receiving erythropoietin for dialysis-associated anemia. Kidney Int. 1989;35(2):712-716.

9. Wexler D, Silverberg D, Sheps D, Iaina A. The importance of correction of anemia with erythropoietin and intravenous iron in severe resistant congestive heart failure. European Journal of Heart Failure. 2003;2:225-230.

10. Goddard AF, James MW, McIntyre AS, Scott BB, British Society of G. Guidelines for the management of iron deficiency anaemia. Gut. 2011;60(10):1309-1316. 
11. Tovbin D, Mazor D, Vorobiov M, Chaimovitz C, Meyerstein N. Induction of protein oxidation by intravenous iron in hemodialysis patients: role of inflammation. Am J Kidney Dis. 2002;40(5):1005-1012.

12. Kalantar-Zadeh K, Regidor DL, McAllister CJ, Michael B, Warnock DG. Time-dependent associations between iron and mortality in hemodialysis patients. J Am Soc Nephrol. 2005;16(10):3070-3080.

13. Lentini P, de Cal M, Cruz D, Chronopoulos A, Soni S, Nalesso F, Zanella M, et al. The role of advanced oxidation protein products in intensive care unit patients with acute kidney injury. J Crit Care. 2010;25(4):605-609.

14. Penne EL, van der Weerd NC, Grooteman MP, Mazairac $\mathrm{AH}$, van den Dorpel MA, Nube MJ, Bots ML, et al. Role of residual renal function in phosphate control and anemia management in chronic hemodialysis patients. Clin J Am Soc Nephrol. 2011;6(2):281-289.

15. Shemin D, Bostom AG, Laliberty P, Dworkin LD. Residual renal function and mortality risk in hemodialysis patients. Am J Kidney Dis. 2001;38(1):85-90.

16. Goetz DH, Holmes MA, Borregaard N, Bluhm ME, Raymond KN, Strong RK. The neutrophil lipocalin NGAL is a bacteriostatic agent that interferes with siderophore-mediated iron acquisition. Mol Cell. 2002;10(5):1033-1043.

17. Kuwabara T, Mori K, Mukoyama M, Kasahara M, Yokoi H, Saito Y, Yoshioka T, et al. Urinary neutrophil gelatinase-associated lipocalin levels reflect damage to glomeruli, proximal tubules, and distal nephrons. Kidney Int. 2009;75(3):285-294.

18. Schmidt-Ott KM, Mori K, Li JY, Kalandadze A, Cohen DJ, Devarajan P, Barasch J. Dual action of neutrophil gelatinase-associated lipocalin. J Am Soc Nephrol. 2007;18(2):407-413.

19. Supavekin S, Zhang W, Kucherlapati R, Kaskel FJ, Moore LC, Devarajan P. Differential gene expression following early renal ischemia/reperfusion. Kidney Int. 2003;63(5):1714-1724.

20. Bolignano D, Coppolino G, Romeo A, De Paola L, Buemi A, Lacquaniti A, Nicocia G, et al. Neutrophil gelatinase-associated lipocalin (NGAL) reflects iron status in haemodialysis patients. Nephrol Dial Transplant. 2009;24(11):3398-3403.

21. Viau A, El Karoui K, Laouari D, Burtin M, Nguyen C, Mori K, Pillebout E, et al. Lipocalin 2 is essential for chronic kidney disease progression in mice and humans.
J Clin Invest. 2010;120(11):4065-4076.

22. Mishra J, Ma Q, Prada A, Mitsnefes M, Zahedi K, Yang J, Barasch J, et al. Identification of neutrophil gelatinase-associated lipocalin as a novel early urinary biomarker for ischemic renal injury. J Am Soc Nephrol. 2003;14(10):2534-2543.

23. Haase M, Bellomo R, Devarajan P, Schlattmann P, HaaseFielitz A, PharmD NGAL Meta-analysis Investigator Group. Accuracy of neutrophil gelatinase-associated lipocalin (NGAL) in diagnosis and prognosis in acute kidney injury: a systematic review and meta-analysis. Am J Kidney Dis. 2009;54(6):1012-1024.

24. Bahmani P, Halabian R, Rouhbakhsh M, Roushandeh AM, Masroori N, Ebrahimi M, Samadikuchaksaraei A, et al. Neutrophil gelatinase-associated lipocalin induces the expression of heme oxygenase- 1 and superoxide dismutase 1, 2. Cell Stress Chaperones. 2010;15(4):395-403.

25. Mori K, Lee HT, Rapoport D, Drexler IR, Foster K, Yang J, Schmidt-Ott KM, et al. Endocytic delivery of lipocalin-siderophore-iron complex rescues the kidney from ischemia-reperfusion injury. J Clin Invest. 2005;115(3):610-621.

26. Witko-Sarsat V, Friedlander M, Capeillere-Blandin C, Nguyen-Khoa T, Nguyen AT, Zingraff J, Jungers P, et al. Advanced oxidation protein products as a novel marker of oxidative stress in uremia. Kidney Int. 1996;49(5):13041313.

27. Bolignano D, Coppolino G, Campo S, Aloisi C, Nicocia G, Frisina N, Buemi M. Neutrophil gelatinase-associated lipocalin in patients with autosomal-dominant polycystic kidney disease. Am J Nephrol. 2007;27(4):373-378.

28. Bolignano D, Coppolino G, Campo S, Aloisi C, Nicocia G, Frisina N, Buemi M. Urinary neutrophil gelatinase-associated lipocalin (NGAL) is associated with severity of renal disease in proteinuric patients. Nephrol Dial Transplant. 2008;23(1):414-416.

29. Hall IE, Yarlagadda SG, Coca SG, Wang Z, Doshi M, Devarajan P, Han WK, et al. IL-18 and urinary NGAL predict dialysis and graft recovery after kidney transplantation. J Am Soc Nephrol. 2010;21(1):189-197.

30. Bolignano D, Lacquaniti A, Coppolino G, Donato V, Campo S, Fazio MR, Nicocia G, et al. Neutrophil gelatinaseassociated lipocalin (NGAL) and progression of chronic kidney disease. Clin J Am Soc Nephrol. 2009;4(2):337344. 